\title{
Desempeño profesional de los decisores/gestores en enfermería para la seguridad del paciente
}

\section{Professional performance of decision-makers / managers in nursing for patient safety}

Lourdes Sherwood Ilizastigui. ${ }^{1}$, Yamilet Portela Lauzurica. ${ }^{2}$, Anabel González Sánchez ${ }^{3 \cdot}$ \& Miguel Rodríguez Curbelo. ${ }^{4}$

Recibido: 10-07-2020 / Revisado: 15-08-2020 /Aceptado: 04-09-2020/ Publicado: 03-10-2020

DOI: $\underline{\text { https://doi.org/10.33262/anatomiadigital.v3i4.1436 }}$

\begin{abstract}
.
Introduction: The nurse from its beginnings has been considered as a social product linked to the art of taking care, the patient's security. As sure practice it is a problem at world level. The nursing agent's professional acting, is an element to consider evaluating the quality of the attention of health that its professionals offer, it is revealed like a necessity to investigate. Objective: to systematize the professional acting of the
\end{abstract}

Resumen. Introducción: La enfermería desde sus inicios se ha considerado como $u$ $\mathrm{n}$ producto social vinculado al arte de cuidar, la seguridad del paciente. Como práctica segura es un problema a nivel mundial. El desempeño profesional del decisor/gestor de enfermería, es un elemento a considerar para evaluar la calidad de la atención de salud que ofrecen sus profesionales, se revela como una necesidad a investigar.

Objetivo:

\footnotetext{
Universidad de Ciencias Médicas, Enfermería, Matanzas, Cuba, sherwood.mtz@infomed.sld.cu https://orcid.org/0000-0002-5147-5589

2 Universidad de Ciencias Médicas, Enfermería, Matanzas, Cuba, yamiletpl.mtz@infomed.sld.cu https://orcid.org/0000-0003-2943-5 7722-

Universidad de Ciencias Médicas, Enfermería, Matanzas, Cuba anabelg.mtz@infomed.sld.cu https://orcid.org/0000-0002-3528-5761

4 Universidad de Ciencias Médicas, Enfermería, Matanzas, Cuba miguelr.mtz@infomed.sld.cu (D) https://orcid.org/0000-0002-7412-7843
} 
nursing managers/decision for the patient's security. Methodology: was carried out a revision in different databases. Infomed, Cochrane, Library, Lilac, CINHAL, Dialnet, PubMed, Scielo, Scopus, they were used for the search the articles referred describers in Spanish language, English and available Portuguese in the portals of selected data that they presented adherence to the thematic one, published among the years 2000 and 2019. The exclusion approaches were the investigations that were repeated in the databases the editorials and the letters to the editor. Results: To support the whole definition with regard to Professional performance and the patient's security they are supported the definitions of Nurse, their functional List, decision-makers / managers sure Care and the patient's Security as a result. Conclusion: as a result of the analysis of the definitions on acting professional facilitated the analysis with a systemic conception of the managers/agent's work in nursing in its professional acting, asì the authors gave its approach of the professional acting in relaciòn to the patient's security.

Keywords: Nursing, patient safety, professional performance, decision makers / managers

\section{Introducción.}

La enfermería desde sus inicios se ha considerado como un producto social vinculado al arte de cuidar, por lo que responde a la necesidad de ayudar a las personas, cuando estas no tienen capacidad suficiente para proporcionarse a sí misma o a las personas, que dependen de ellas, la calidad y cantidad de cuidados para mantener la vida, identificar los problemas de salud y 
las necesidades reales y/o potenciales de la persona, familia y comunidad que demandan cuidados, que generen autonomía o dependencia.(LeònC,2006, Raille,2015)

En Enfermería, la tendencia de la profesión ha girado en torno a la implantación de estrategias de mejoramiento continuo y satisfacción de las necesidades del individuo, familia y colectivos. Es por ello que para garantizar la calidad en el cuidado de la salud y de la vida del ser humano, los profesionales de Enfermería deben responder con un nivel alto de excelencia en su ejercicio, un alto grado de satisfacción del paciente con riesgo mínimo para él, y que los haga sentir seguros, en la Organización Mundial de la Salud (OMS). La 55 Asamblea Mundial de la Salud, celebrada en Ginebra en el 2002 se aprobó la resolución WHA 55.13 que instó a los estados miembros a prestar la mayor atención posible al problema de la seguridad del paciente y a consolidar sistemas científicos necesarios para mejorar la seguridad y calidad de la atención en salud OMS'(OMS,A55/13)

La seguridad del paciente como práctica segura es un problema a nivel mundial, lo que significa que no se facilitan servicios de calidad a los usuarios, lo que aumenta la probabilidad de producir efectos no deseados en la salud de los individuos. Los autores Cristián Rocco \& Alejandro Garrido, definen a la seguridad del paciente como aquellos procesos elementales utilizados para impedir daños u alteraciones a los pacientes durante la asistencia médica, la seguridad es la parte básica para brindar un servicio de calidad en salud para evitar cualquier complicación o condición durante la actividad clínica. (Criastian, 2017) Los autores refieren que según la Organización Mundial de la Salud que la seguridad del paciente en un problema de salud en el mundo y representa el puesto número 14 de las causas de morbi mortalidad, basado en la "Incidencia de efectos adversos relacionados con la asistencia hospitalaria" (OMS, 2019, Comisión E, 2014,CIE, 2018)

Los deisores/gestores de enfermería deben practicar y hacer parte de su quehacer diario las herramientas de contribuir en alcanzar los objetivos institucionales, se debe entregar a los profesionales las herramientas indispensables y necesarias para poder alcanzar la calidad esperada en la gestión, siempre considerar estas habilidades dentro de la praxis ,ser ejemplo de las enfermeras asistenciales. (Soares M,Resck,ZCamelo,2016 )

Es necesario encargarse de diseño de políticas de salud, generar mecanismos de planificación de recursos humanos acordes a las demandas actuales, proveer de dotación de personal equitativo y calificado en la búsqueda de la satisfacción del paciente, si el desempeño profesional del gestor logra con calidad se está ante un trabajo de excelencia. (Colectivo de autores,2017,GunawanJ,et,al,2018,Guedes Dos Santos, 2009, González J,2016 )

El desempeño profesional ha sido ampliamente estudiado en Europa, Norteamérica, Japón y Australia ,países en los cuales las investigaciones se han enfocado en el paradigma de las competencias profesionales y su relación con el desempeño.( Crawford CL,et,al 
,2017,SànchezM,2010,Moran BJ,2016) Por tanto, aunque en los servicios los procesos son los mismos, el ambiente no es igual, depende de cada una de las enfermeras, de su esencia del conocimiento que tengan, de la motivación y de las relaciones del equipo de salud .

El desempeño profesional de enfermería, elemento a considerar para evaluar la calidad de la atención de salud que ofrecen sus profesionales, se revela como una necesidad a investigar en esta área. Algunos autores Santiesteban ML, Santamaria (ArizaC,DazaR,2005 'AñorgaJ,2002), en su sistematización de sus investigaciones han tendido a asociar el concepto de desempeño con capacidad, competencia, modo de actuación y proceso .

Los autores le dan relevancia a la Dra. Cs Julia Añorga en el año 2012 , porque ha sido una estudiosa del desempeño profesional que identifica una categoría como punto de partida para el proceso del mejoramiento humano, ya que lo define como un proceso consciente que desarrollan los hombres en un escenario habitual donde se realizan sus labores enmarcado en un sistema de normas y procedimientos que deben cumplirse, en un resultado satisfactorio.(AñorgaJ,2012)

Atemperamos las definiciones de desempeño profesional con respecto a la seguridad del paciente y los autores definen; Él desempeño profesional para la seguridad del paciente tiene un carácter de proceso que permite identificar acciones propias de los profesionales de enfermería, que atesoran las normas y procedimientos donde se debe lograr una satisfacción del paciente con la calidad requerida en función de evitar eventos adversos. Los estudios que priorizan el desempeño profesional y su conocimiento sobre seguridad del paciente coinciden en colocar entre las más importantes: las dimensiones técnico-profesional, superación, investigación y progresión de una falla o la producción de daño al paciente, con el propósito de prevenir o mitigar sus consecuencias en el comportamiento.( Rodríguez G, Hechavarria , Y,2018, - Merrill KC,2015)

Los autores conciben la función asistencial como rectora de las demás funciones, mediante su cumplimiento el gestor debe integrar las habilidades específicas de la profesión, impartir docencia a su nivel mientras se desempeña en su trabajo, con ética y respeto

Otros autores como Valcárcel 2009, Sixto 2014, Ramos 2017, y González 2017 proponen Estrategias de Superación, los autores persiguen ese fin ya que es una fuente de adquirir conocimientos para que el gerente/gestor este actualizado. El objetivo de este estudio es sistematizar el desempeño profesional de los decisores/gestores de enfermería para la seguridad del paciente.

\section{Metodologia.}

Se realizó una revisión bibliográfica sistemática para desarrollar un análisis crítico reflexivo del contenido de documentos, donde se consideraron artículos originales y de revisiones disponibles en INTERNET, así como de tesis de maestrías y doctorado. 
Tras la identificación de los estudios pre-seleccionados se llevó a cabo la lectura de los títulos de las publicaciones, resumen y palabras clave, comprobando la pertinencia con el estudio, debiendo estar adherido a la temática abordada. Se encontraron más de 100 artículos, se preciso la búsqueda se seleccionaron 114 artículos, se seleccionaron las publicaciones centradas en el desempeño profesional (40) ,cuidados(20), gestión(23), seguridad del paciente(40), tesis doctorales (10), de maestrías (5) y de profesionalización(5) y documentos que rigen la seguridad del paciente en el contexto mundial y nacional así se releva el desarrollo científico de la enfermería, sirvió de referencia al estudio realizado .

La estrategia de búsqueda que se estableció fue la utilización de las palabras clave o descriptores, conectados por intermedio del operador booleano AND. Las palabras clave utilizadas fueron "desempeño profesional", "enfermería ", decisores ,"gestores, "seguridad del paciente ", siendo estas identificadas a través de DECs o de MeSH. De esta forma, fueron utilizados para la búsqueda los artículos referidos descriptores en idioma español, inglés y portugués. La búsqueda fue realizada en las bases de datos Infomed ,Cochrane ,Library, Lilac, CINHAL, Dialnet, PubMed, Scielo ,Scopus. de (rango de meses o años en los cuales realizaste la búsqueda)

En la selección de los artículos los criterios de inclusión se eligieron : Artículos en español portugués e inglés disponibles en los portales de datos seleccionados que presentaban adherencia a la temática, publicados entre los años 2000 y 2019 que presentaran de manera clara la metodología o referencial teórico seleccionado. Los criterios de exclusión fueron las pesquisas que se encontraron repetidas en las bases de datos los editoriales y las cartas al editor.

\section{Resultados.}

En párrafos anteriores conceptualizamos el desempeño profesional para la seguridad del paciente como un proceso que permite identificar acciones propias de los profesionales de enfermería, que atesoran las normas y procedimientos donde se debe lograr una satisfacción del paciente con la calidad requerida en función de evitar eventos adversos.

En vista al logro de ese desempeño profesional para la seguridad del paciente. Se hace alusión al cuidado de enfermería, se afirma que "tiene como fin dar cuidado integral de salud a la persona, familia, la comunidad y a su entorno; ayudar a desarrollar al máximo los potenciales individuales y colectivos, para mantener prácticas de vida saludables que permitan salvaguardar un estado óptimo de salud en todas las etapas de la vida". (DiVora,2004, LagoueyteMI,2015) El desempeño de los decisores/gestores de enfermería está basado en la ejecución de actividades profesionales y refleja el conocimiento, habilidades y destrezas de los mismos, donde el liderazgo, la autonomía profesional, la responsabilidad, la toma de decisiones, la identidad profesional, son elementos 
fundamentales para optimizar la prestación de los servicios en enfermería. (Guedes dos Santos,et,al,2009, Gonzales García J,2016 )

Para respaldar toda la definición operacional de términos relacionamos en los párrafos siguientes

Enfermería. Para este trabajo de investigación se entiende por enfermería todo personal del área de la salud que ha recibido formación en el área de enfermería auxiliar, técnica o profesional en una institución de educación vocacional, técnica y/o superior, reconocida nacionalmente y que cuenta con certificación o título profesional. (LeònC,2006, Raille,2015)

Rol funcional de enfermería. Es un conjunto de competencias, funciones, actividades o tareas que desarrolla el profesional de enfermería en un área específica de actuación o desempeño.( Crawford CL,2017, Sánchez M ,Z 2010)

Decisor: Es el encargado de realizar la selección de alternativas de la mejor manera, en función de sus objetivos, se traduce como aquel que toma las decisiones dentro de los aspectos llamados importantes ya sea en el caso de la salud, educación u otro dentro de las funciones del estado, los decisores son también llamados "tomadores de decisiones"( Real academia española ,2014)

Gestor: Es el individuo que hace referencia a la acción y al efecto debe gestionar o de administrar. Se trata, por lo tanto, de la concreción de diligencias conducentes al logro de un negocio o de un deseo cualquiera. La noción implica además acciones para gobernar, dirigir, ordenar, disponer u organizar. Real academia española

Cuidado seguro "es aquel que además de cumplir con las necesidades y expectativas del paciente, al ser desarrollado alcanza un estado de logro que beneficia en todos los sentidos a la persona atendida .(OMS, A55/13,2002,Martínez E,2018,LagoueyteMI,2015)

Seguridad del paciente. Es el conjunto de elementos estructurales, procesos, instrumentos y metodologías basados en evidencias científicamente probadas, que propenden por minimizar el riesgo de sufrir un Evento adverso en el proceso de atención de salud o de mitigar su consecuencia. (OMS, A55/13,2002, OMS, 2018, NC-ISO/IEC 31010:2015, AgraVarela, 2017)

\section{Conclusiones.}

- Se realiza un criterio concluyente que las definiciones sobre desempeño profesional posibilitaron el análisis con una concepción sistémica de la labor del gestor en Enfermería en su desempeño profesional, así es como la relación con la seguridad del paciente, está enmarcada en el rol de enfermería, el cuidado seguro incluyendo la seguridad del paciente como colofón de ese desempeño profesional para llegar a la solución de los problemas. 
- La presente investigación cobra gran importancia en la medida que la formación de los enfermeros, en general, contempla de manera muy significativa los aspectos de gestión y toma de decisión de los servicios de salud y de Enfermería.

- Los autores en su conceptualización de desempeño profesional para la seguridad del paciente reviste la importancia que los profesionales en enfermería están llamados a comprender los distintos lineamientos de las políticas públicas para orientar la toma de decisiones en los diferentes roles: asistencial, docencia, investigativo $\mathrm{y}$ administrativo.

\section{Referencias bibliográficas.}

Agra-Varela Y. Seguridad del paciente en el pasado, presente y futuro de las organizaciones sanitarias. Un desafío para las enfermeras. Enfer Clin. [Internet]. 2017; Recuperado de https://www.elsevier.es.revenfermeriaclinica:http://dx.doi.org/10.10

Ariza C, Daza de Caballero R. Calidad del cuidado de enfermería al Paciente hospitalizado en un hospital de tercer nivel en Bogotá. 2005 Recuperado de: http://encolombia.com/medicina/enfermeria/Enfermeria8305- Calidad.

Añorga J. La producción intelectual: Proceso organizativo y pedagógico. La Habana, Cuba: Editorial Universitaria; 2002. p. 50-60.

Añorga J. La Educación Avanzada y el mejoramiento profesional y Humano. Tesis en opción al grado científico de Doctora en Ciencias .Segundo doctorado. La Habana, Cuba: Universidad de Ciencias Pedagógicas "Enrique José Varona"; 2012

CIE. Consejo Internacional de Enfermería aprueba la Declaración de Tokio sobre la seguridad del paciente [Internet]. 2018. Recuperado de: :http://www.icn.ch/imágenes/documents/2018_Tokyo_Decla_Ration_es Pdf

Comisión Europea. Salud Pública. Seguridad de los pacientes. Bruselas : Comisión Europea; [Internet]. 2014.;Recuperado de: http://ec.europa.eu/health/patient_safety/policy/index_es.htm

Crawford CL, Omery A, Spicer J. An Integrative Review of 21st-Century Roles, Responsibilities, Characteristics, and Competencies of Chief Nurse Executives: A Blueprint for the Next Generation. Nurs Adm Q. 2017;41(4).

Cristián Rocco, \& Alejandro Garrido. Seguridad del paciente y Cultura de seguridad. Revista Médica Clínica Las Condes, 28(5), 785-795. Recuperado de:https: //doi.org/10.1016/j.rmclc.2017.08.006

DI VORA, MERCEDES, El desempeño del Maestro y su actuación, Editorial Pueblo y Educación, 2004. p. 54.

Gonzales García J. Competencias de los gestores enfermeros: Una revisión sistemática [Internet]. Universidad de Léon; 2016. Disponible en: https://buleria.unileon.es/bitstream/handle/10612/6865 
Gunawan J, Aungsuroch Y, Fisher ML. Factors contributing to managerial competence of first-line nurse managers: A systematic review. Int J Nurs Pract [Internet]. 2018;24. Disponible en: https://doi.org/10.1111/ijn.12611

Guedes dos Santos L, Garlet ER, Dias da Silva Lima MA. Revisão sistemática sobre a dimensão gerencial no trabalho do enfermeiro no âmbito hospitalar. Rev Gaúcha Enferm. 2009;30(3):525-32.

Lagoueyte Gómez MI. El cuidado de enfermería a los grupos humanos.Rev Univ Ind Santander Salud. 2015 [acceso: 13/04/2018];47(2):20 Recuperado desde: http://www.scielo.org.co/scielophp?script

León Román CA. Enfermería ciencia y arte del cuidado. Rev Cubana Enfermer[Revista en Internet]. 2006; 22(4): [aprox. 5 p.]. Recuperado desde: http://scielo.sld.cu/scielo.php?script=sci_arttext\&pid

Manual de admistraciòn y gestión de enfermería .Colectiv de autores Capitulo 5La Habana .Editorial Ciencias Mèdicas.2017

Martínez Sariol Elsa. Estrategias de superación para el desarrollo de competencias profesionales especifica en la atención de Enfermería al neonato crìtico. [Tesis doctoral].2018.Recuperado desde: http://tesis.sld.cu

Merrill KC. Estilos de liderazgo y seguridad del paciente: implicaciones parlos gerentes de Enfermería. J NursAdm. [Internet]. 2015.45(6): 319-24. Disponible en:https://www.ncbi.nlm.nih.gov.doi.org/

Moran-Barrios J. La evaluación del desempeño o de las competencias en la práctica clínica. 1a Parte: principios y métodos, ventajas y desven-tajas. Educ Med. 2016; 17(4): http://dx.doi.org/10.1016/j. edumed. 2016

15-96. NC-ISO/IEC 31010:2015. Gestión del riesgo. Técnicas de aprecia-

ción del riesgo. Oficina Nacional de Normalización NC. [Internet]. 2016 La

Habana, Cuba. Recuperado desde: WWW.NC.Cubaindustria.cu

OMS, A55/13.. Calidad de la Atención: seguridad del paciente. Informe de la Secretaría. (23 de marzo de 2002).Recuperado desde: http://www.who.int/patientsafety

Organización Mundial de la Salud Reto mundial en pro de la Seguridad del paciente. Una atención limpia es una atención más segura. World Healt

Organization. (2018) Disponible en: http://www.who.int/patientsafety

Raille Alligood.Modelos y teorías en enfermería.8ta edición Barcelona

Elseiver 2015. Real academia española decisor.html, Recuperado desde: https://www. google.com http://necedidadesdeldecisor.blogspot.com/2014/11/que-es-un-Real academia española ,Recuperado desde: https://economipedia.com/definiciones

Rodríguez García, Graciela 1 Hechavarria López, Yelaine 2 Gestión del cuidado a pacientes desde la formación de dirigentes de enfermería Octubre 2017 - Mayo 2018 X V I II Congreso de la Sociedad cubana de enfermería Recuperado desde : http://scielo.sld.cu/scielo.php?script\&pid 
Vol. 3, N4, p. 44-53, octubre-diciembre, 2020

Sánchez Mirtha Zuleyka. Desempeño laboral de los gerentes de Enfermería y la motivación de las enfermeras de cuidad directo. 7/05/2010 | Enfermería ,Gestión ||

Soares Inácio M, Resck Rodrigues Z, Camelo Henriques SH, Terra de Souza F. Gestión de recursos humanos y su interfaz en la sistematización de la asistencia de enfermería. Enfermería Glob [Internet]. 2016;42:341- 52. Recuperado desde: http://scielo.isciii.es/pdf/eg/v15n42/administracion3.pdf 


\section{PARA CITAR EL ARTÍCULO INDEXADO.}

Sherwood Ilizastigui, L., Portela Lauzurica, Y., González Sánchez , A., \& Rodríguez Curbelo, M. (2020). Desempeño profesional de los decisores/gestores en enfermería para la seguridad del paciente. Anatomía Digital, 3(4), 44-53. https://doi.org/10.33262/anatomiadigital.v3i4.1436

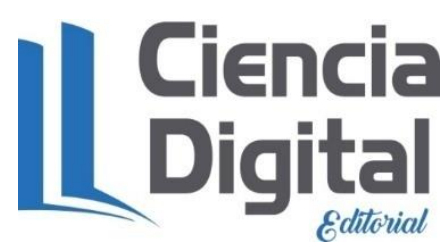

El artículo que se publica es de exclusiva responsabilidad de los autores y no necesariamente reflejan el pensamiento de la Revista Anatomía Digital.

El artículo queda en propiedad de la revista y, por tanto, su publicación parcial y/o total en otro medio tiene que ser autorizado por el director de la Revista Anatomía Digital.
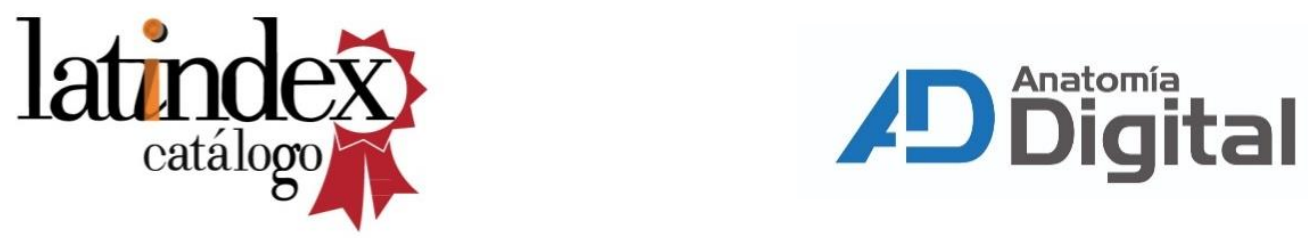